\title{
Optical amplification enhancement in photonic crystals
}

\author{
R. Sapienza, ${ }^{1, *}$ M. Leonetti, ${ }^{1}$ L. S. Froufe-Pérez, ${ }^{1}$ J. F. Galisteo-López, ${ }^{1}$ C. Conti, ${ }^{2}$ and C. López ${ }^{1, \dagger}$ \\ ${ }^{1}$ Instituto de Ciencia de Materiales de Madrid (CSIC) and Unidad Asociada CSIC-UVigo, Cantoblanco, E-28049 Madrid, Spain \\ ${ }^{2}$ Research Center INFM-CNR, clo Universitá di Roma Sapienza, I-00185 Roma, Italy
}

(Received 16 September 2010; published 4 February 2011)

\begin{abstract}
Improving and controlling the efficiency of a gain medium is one of the most challenging problems of laser research. By measuring the gain length in an opal-based photonic crystal doped with laser dye, we demonstrate that optical amplification is more than twenty-fold enhanced along the $\Gamma-K$ symmetry directions of the facecentered-cubic photonic crystal. These results are theoretically explained by directional variations of the density of states, providing a quantitative connection between density of the states and light amplification.
\end{abstract}

DOI: 10.1103/PhysRevA.83.023801

PACS number(s): 42.70.Qs, 42.55.Tv, 42.70.Hj

\section{INTRODUCTION}

The study of unconventional lasing is a novel and active field of research. Mirrorless, micron-sized, and threedimensional lasers, in the form of random lasers [1], microsphere and microdrop lasers [2], photonic crystal ( $\mathrm{PhC})$ lasers [3], and chaotic-cavity lasers [4] recently have been proposed as more sophisticated alternatives to standard lasers made of macroscopic mirrors and Fabry-Pérot cavities.

Artificially engineered photonic materials, with nanoscopic features and topologies ranging from order to disorder, allow for superior control of light modes, dispersion, and gain [5]. Photonic structures can control and tune the directionality and spectral extent of the emitted light without relying on light absorption, allowing for efficient light sources with smarter functionalities.

While the research into two-dimensional (2D) PhC lasing can exploit high-quality and low-volume microcavities [6], in three dimensions (3D) PhC lasing so far is based only on the large light-matter interaction that can be achieved by extended Bloch modes, at band edges [7], or at high-energy flat bands [8]. These modes effectively confine the light in the active medium and increase the amplification and the gain extraction from large bulk volumes [9]. A similar confinement, but originated by multiple scattering, is responsible for random lasing of diffusive modes [1]. The first pioneering study of gain in $\mathrm{PhC}$ has demonstrated a directional modification of the gain spectral profile, attributed to band-edge effects [10]. Enhanced optical gain was also extrapolated from a decreased lasing threshold at the $L$-pseudogap edges of an opal- $\mathrm{TiO}_{2}$ composite containing quantum dots [11].

The lasing wavelength selection and the decrease of the lasing threshold in PhC lasers often has been claimed to be connected to spectral maxima of density of states [9], but quantitative evidence of the directional nature of the gain length due to the dependance of the density of the states on the crystallographic direction in which the amplification grows has not been reported so far. Demonstrating this link is of paramount importance not only for the future developments

\footnotetext{
${ }^{*}$ Current address: ICFOInstitut de Ciencies Fotoniques, Mediterranean Technology Park, E-08860 Castelldefels (Barcelona), Spain.

${ }^{\dagger}$ www.luxrerum.org; cefe@icmm.csic.es
}

of nanostructured laser devices but also as a starting point for several fundamental investigations ranging from quantum information to slow light phenomena.

\section{GAIN MEASUREMENT}

In this article, we report a detailed measurement of the gain length in opal-based photonic crystals doped by rhodamine 6G. The measured gain length has a six-fold symmetry, takes values up to $\sim 500 \mathrm{~cm}^{-1}$ along the $\Gamma-K$ directions in reciprocal space, and decreases to values below measurable levels of $\sim 20 \mathrm{~cm}^{-1}$ far from the high symmetry directions. We compare these findings to the directional density of states, which has been predicted to be responsible for the increased gain.

The samples under study are thin-film artificial opals consisting of a face-centered-cubic (fcc) matrix of polystyrene spheres (PS, refractive index, $n=1.59$, diameter $d=512 \pm$ $15 \mathrm{~nm}$ ) bulk-doped with rhodamine $6 \mathrm{G}$ with an emission centered around $\lambda=574 \mathrm{~nm}$ (from Duke Scientific). The fabrication process, that is, the vertical deposition method [12] fixes a well-defined growth direction (that of the evaporating meniscus), which correspond to one of the $\Gamma-K$ directions in reciprocal space

Figure 1 shows transmission and reflection spectrum from the sample [in panel (a)] as well as two scanning-electronmicroscope (SEM) pictures of the top of sample [panel (b)] and its side [panel (c)]. While a band gap is visible at around $1200 \mathrm{~nm}$, the dye emission indicated by the gray area are in the high energy peaks of the spectrum around $574 \mathrm{~nm}$.

The optical gain in the dye-doped 3D artificial opals can be extracted by employing the stripe-length technique $[13,14]$. The emission intensity and amplification are measured as a function of the length of the illuminated area on the sample. In Fig. 2(a), a sketch of the measurement configurations used for the experiment is presented, while Fig. 2(b) reports the curves from which the amount of optical amplification is extracted. The spontaneous emitted photons, traveling in the direction $z$ of the illuminated stripe, undergo an exponential amplification of the intensity with respect to the excitation length $z$ as they pass through an area of population inversion. This can be quantified by the gain coefficient, the most important actor in light amplification and lasing, which is a robust quantity, 

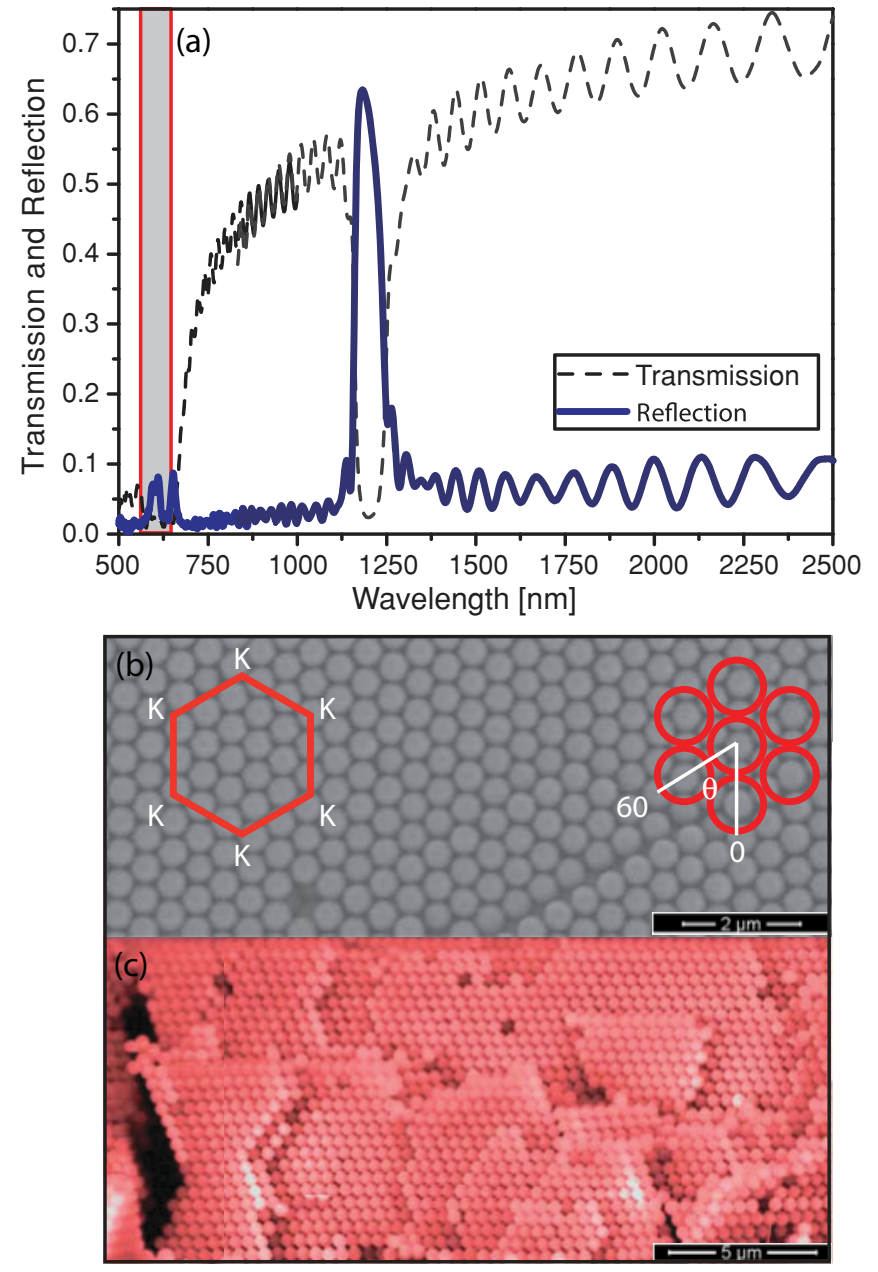

FIG. 1. (Color online) (a) Photonic crystal transmission and reflection spectra. The shaded region indicates the gain curve of the dye. (b) SEM image of the top of the sample with a sketch of the crystallographic directions and the real space arrangement. The hexagon indicates a cut of 3D Brillouin in the plane (111), and (c) SEM image of the side of the sample, corrected with the real color of the dye-doped spheres.

independent of the sample thickness (in absence of pump depletion) and of the edge coupling of the light from the stripe to the detector. The output intensity $I(z)$ collected at one edge of the stripe is the sum of spontaneous and stimulated emission and has the well-known spatial dependence [13]

$$
\begin{gathered}
I(z)=I_{\mathrm{SE}}[\exp (G z)-1], \\
G=g-\ell_{a}^{-1}-\ell_{s}^{-1},
\end{gathered}
$$

where $I_{\mathrm{SE}}$ is the spontaneous emission (SE) collected by the detector, $g$ is the gain (including confinement effects), $\ell_{a}$ is the absorption length, and $\ell_{s}$ is the scattering mean free path. The quantity $G$, refereed to as the net gain, is directly obtained from stripe-length measurements. Equation (1a) holds until gain saturation is reached, which occurs for stripe lengths larger than around 150-200 $\mu \mathrm{m}$, in our case.

In our experiment, the excitation is carried out by illuminating the sample along the $\mathrm{PhC}$ [111] direction (as

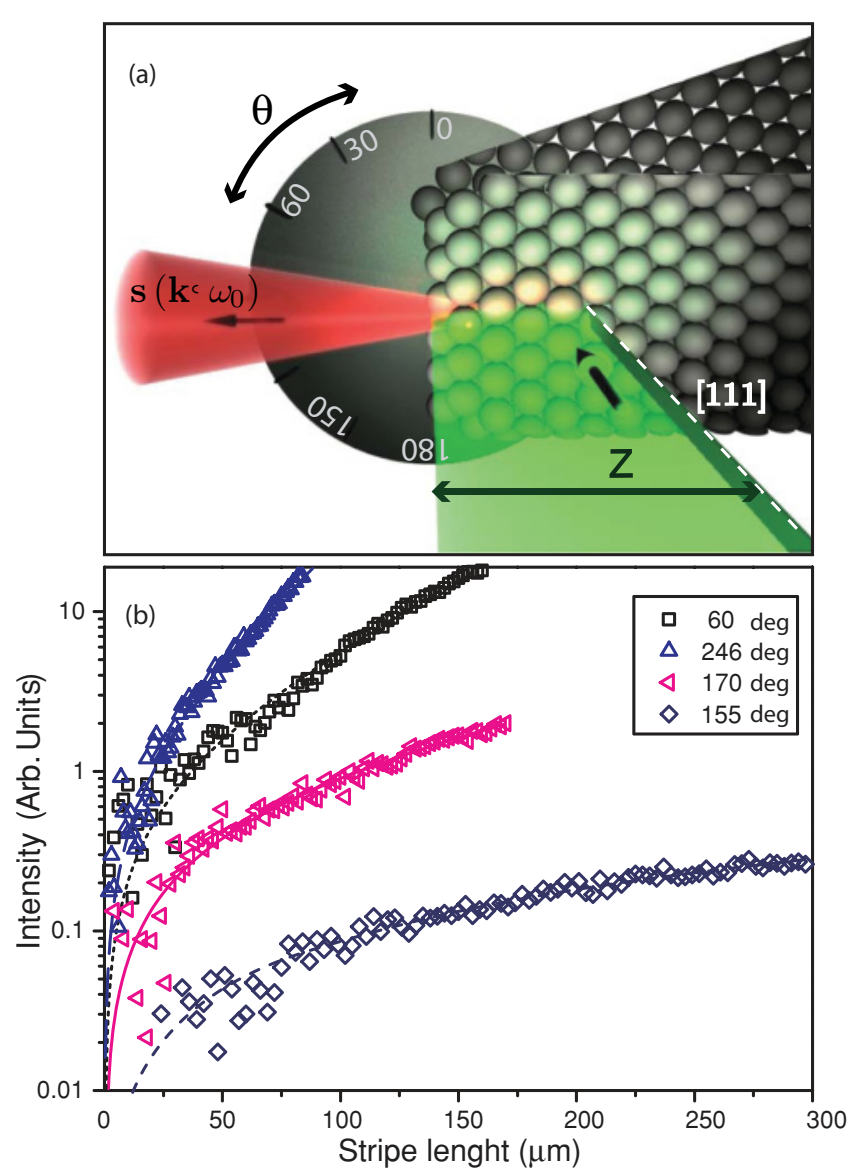

FIG. 2. (Color online) (a) Sketch of two configurations for measurement of the $G$ parameter. The green laser pump is incident along the $\langle 111\rangle$ direction, and the red emission in the direction $\mathbf{s}\left(\mathbf{k}, \omega_{0}\right)$ is collected on a side by a miniature spectrometer. The $\mathrm{PhC}$ is rotated continuously in the plane (111) orthogonal to the [111] direction to explore the angle $\theta$ dependence of the emission. The illumination stripe has a variable length $z$. (b) Intensity of the emitted light, as a function of the length, collected at different angles. The symbols correspond to the emissions as follows: black squares, $60 \mathrm{deg}$; blue triangles, $246 \mathrm{deg}$; red triangles, $170 \mathrm{deg}$; and blue diamonds, $155 \mathrm{deg}$. The continuous lines represent the fits obtained by using the equation reported in the experimental section.

in Fig. 2(a)) with a Q-switched Nd:Yag laser, 9-ns pulses, and $10-\mathrm{Hz}$ repetition rate, at a wavelength of $532 \mathrm{~nm}$. The laser beam has a stripe of thickness $\sim 20 \mu \mathrm{m}$ obtained by focusing the laser source with a cylindrical lens of $75 \mathrm{~mm}$ focal length. Measurements of the dependence of $I$ as a function of $z$ has been performed by cutting the stripe with a blade moved automatically with micrometer accuracy. A miniature fiber-coupled spectrometer is used to efficiently collect the light along the stripe direction, as described in Ref. [14].

By rotating the stripe relative to the in-plane direction s, various crystallographic directions, including the highsymmetry ones, can be explored. In Fig. 2(b), the dependence of the collected fluorescence intensity on the stripe length $z$ for various different orientations of the photonic crystal is shown. Emissions for light propagating along two $\Gamma-K$ 
crystal directions are presented (60 and $240 \mathrm{deg}$ ), as well as an intermediate position $(170 \mathrm{deg})$. A nonsymmetrical direction is probed too $(155 \mathrm{deg})$.

\section{BAND AND ISOFREQUENCIES CALCULATION}

The clear dependence of the fluorescence intensity on the propagating angle in the crystal results from the photonic crystal band structure, which shows an higher degree of complexity with respect to an homogenous medium. It is plotted in Fig. 3 along the directions $\Gamma-K, K-L$, and $L^{\prime}-\Gamma$, which are the ones explored when rotating the sample around the direction [111] normal to its surface. The shaded area indicates the active region of the dye where high-order diffractive modes exist that are excited when rhodamine is pumped by a frequency doubled Nd:Yag laser (wavelength $532 \mathrm{~nm}$, green line in the figure). Note that $L^{\prime}$ is not a high-symmetry point equivalent to $L$ but the midpoint of the experimental trajectory along the hexagonal facet of the Brillouin zone.

From the full 3D band structure (calculated by preconditioned conjugate-gradient minimization of the block Rayleigh quotient in a plane-wave basis, using a freely available software package [15]), the isofrequency surface and group velocities are obtained. In Fig. 4, each panel shows the isofrequency of an individual band at the working frequency, colored as in Fig. 3. For the sake of clarity, only half the isofrequency, cut along a plane perpendicular to the [111] direction, is presented.

An homogenous media would exhibit a circular (resulting from the section of a sphere) isofrequency contour; in a photonic crystal, a rich structure with a nonconstant cur-
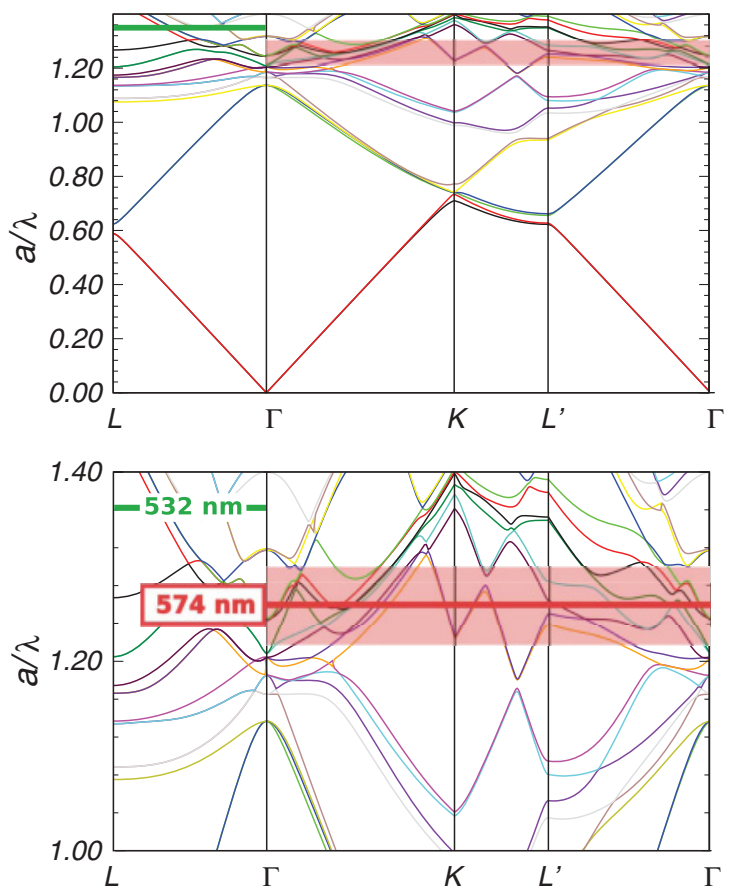

FIG. 3. (Color online) Full (upper panel) and enlarged (lower panel) band diagram. The shaded (red) region indicates the gain curve of the dye centered at $\lambda=574 \mathrm{~nm}$, while the green line at $\lambda=532 \mathrm{~nm}$ represents the pump laser wavelength.
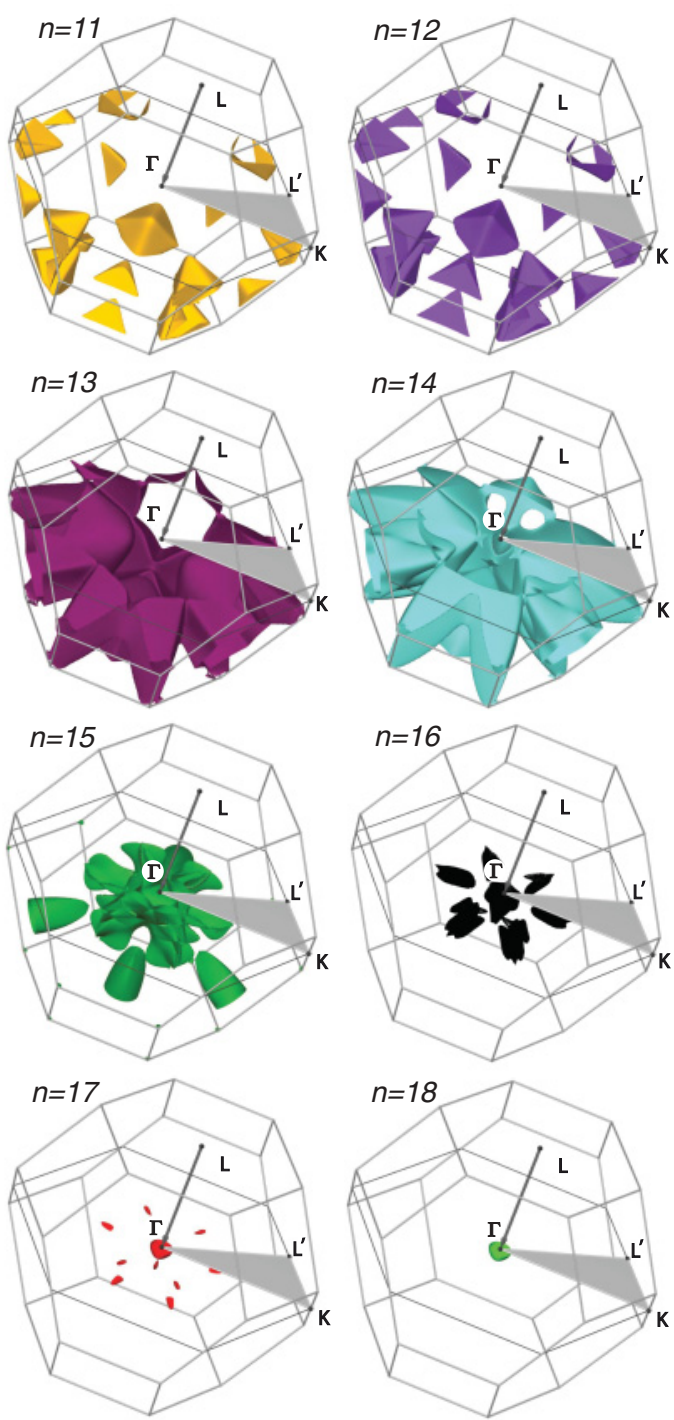

FIG. 4. (Color online) Each of the eight panels represent one isofrequency surface section, for the relative band touching the rhodamine emission at $\lambda=574 \mathrm{~nm}$, cut in the plane perpendicular $(\perp)$ to the $\langle 111\rangle$ direction and parallel $(\|)$ to the sample surface, which is the plane of study. The color code refers to bands on Fig. 3. The relevant symmetry directions are shown by the capital letters.

vature is visible, which results from contributions of eight different bands around the emission energy. Each point in the isofrequency surface identifies a wave vector $\mathbf{k}$, for which the direction of propagation of the energy $\mathbf{s}$ is given by the gradient of the surface.

\section{GAIN AND DOS}

By fitting the data in Fig. 2(b) to Eq. (1), the net gain $G$ can be extracted and plotted as a function of the stripe direction as in Fig. 5. $G$ is affected by the variations of group velocities and varies as a function of the probed crystallographic direction. The angle $\theta=0$ corresponds to the configuration in which the stripe is aligned with the $\Gamma-K$ direction corresponding to the opal growth axis. A large variation of $G$ is visible and 


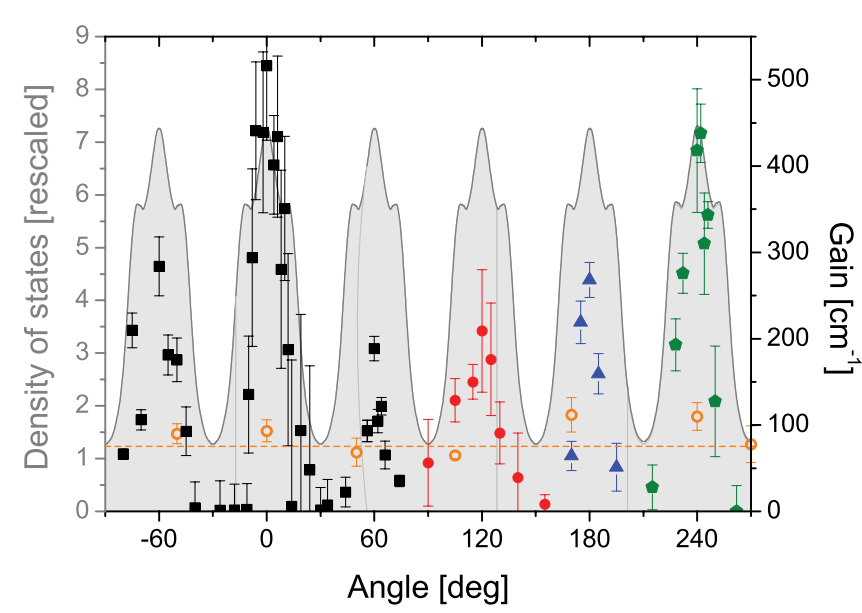

FIG. 5. (Color online) Measured gain in a $\mathrm{PhC}$ as a function of the excitation angle. The dashed (orange) line corresponds to the homogenous reference sample. The emission from the $\mathrm{PhC}$ is presented by the solid symbols, while the gain of the reference sample is denoted by the empty (orange) circles. Different symbols refer to measurements performed after a 90-deg sample rotation. The curve above the shaded region denotes the DOS. $\Gamma-K$ directions occur every $60 \operatorname{deg}$ from $\theta=0 \mathrm{deg}$.

shows maxima along the six $\Gamma-K$ directions (every 60 deg from $\theta=0 \mathrm{deg}$ ) following a C6 symmetry.

The minima measured along less-symmetrical directions reach values that are limited to $\sim 20 \mathrm{~cm}^{-1}$ by the measurement sensitivity: more than 20-fold variation is observed. Differently colored symbols in Fig. 5 correspond to measurement sets that have been performed by rotating the sample $90 \mathrm{deg}$. Thus, each set of data is obtained on a different side of the sample whose good quality-specifically, the homogeneous dye distribution-allows us to retrieve maxima of $G$ corresponding to $\Gamma-K$ directions. Differences in the absolute values of the maxima of $G$ (ranging from 500 to $200 \mathrm{~cm}^{-1}$ ) are due to imperfections resulting from intrinsic disorder and residual inhomogeneities of the samples.

In order to prove that the observed effect depends on the regular disposition of the spheres, a reference sample has been measured too: the same PhC sample after melting by keeping it at $100{ }^{\circ} \mathrm{C}$ overnight. This temperature is enough to deform the PS spheres while keeping the film shape (as confirmed by SEM inspection) without degrading the dye molecules. The reference sample has a dye molecule density $35 \%$ higher than the regular sample, as it is a compact film as compared to the opal $\mathrm{PhC}$, whose filling fraction is 0.74 . The measured gain of $99 \pm 20 \mathrm{~cm}^{-1}$ in the reference is now independent of the excitation angle. The dashed (orange) line in Fig. 5 shows the reference sample gain multiplied by 0.74 to account for the dye molecule density increase.

Since it would be very difficult to obtain analytical results on a photonic structure with a degree of complexity such as that of Fig. 4, we compared our results to the numerically calculated directional density of the states $\rho(\mathbf{s}, \omega)$, that is, the density of modes with Poynting vector pointing in the stripe direction, given by contribution of modes with any $k$ vector in the 3D space. Results are shown in Fig. 5 as the curve above the shaded region.
The extraordinary increase in optical amplification and $G$ shown in Fig. 5, as predicted by Sakoda $[9,16]$, is connected to the variation of the density of states $\rho(\mathbf{s}, \omega)$ in the direction $\mathbf{s}$ of the stripe. The number of available optical states in a given direction, that is, for a given Poynting vector parallel to $\mathbf{s}$, determines the emission probability for the excited dye molecules. A higher value of $\rho(\mathbf{s}, \omega)$ corresponds to a higher emission probability in the $(\mathbf{s}, \omega)$ mode and therefore a larger gain for photons travelling in the $\mathbf{s}$ direction. The shaded region in Fig. 5 shows the variation of $\rho(\mathbf{s}, \omega)$ along the various directions in the (111) plane. The theoretical curve in Fig. 5 has been rescaled by the ratio of the computed value of $\rho(\mathbf{s}, \omega)$ for an homogenous medium to the measured gain in the reference melted opal.

In general cases, also scattering and absorption can vary with the angle. Far from the dye absorption curve, the opal absorption length has been measured to be $\ell_{a} \sim 10 \mathrm{~m} \mathrm{[17],}$ which is therefore negligible for the system under study. The scattering mean free path $\ell_{s}$ can present large variations with $\rho(\mathbf{s}, \omega)$, as studied in Ref. [18]. For lower energy and similar opals, it has been measured to be in the range of 100$500 \mu \mathrm{m}$. This value is larger but comparable to the gain length $\ell_{g}=G^{-1}$, which is here in the range 20-200 $\mu \mathrm{m}$. Large scattering is responsible for only a decrease of the amplification and cannot explain the large increase in $G$ that we have measured. If the variations of $\ell_{g}$ were governed by $\ell_{s}$, then the value of $\ell_{s}$ should be smaller than $\ell_{g}$ and no net gain could be measured. In our case, scattering losses reduce the measured gain in Fig. 5 and set a minimum value of $G$ that can be measured in our experiment $\left(\sim 20 \mathrm{~cm}^{-1}\right)$.

In conclusion, we have presented an observation of a six-fold increase of the gain in an opal photonic crystal, as compared to the homogeneous film, and a more than 20-fold variation of $G$ between $\Gamma-K$ and less symmetrical directions, in the same photonic crystal. We explain this enhancement as due to a large increase of the density of the available modes when exciting around the $\Gamma-K$ direction. Large variations of the gain in $\mathrm{PhC}$ show the impact of the tailored density of states on light generation and amplification and open the way to enhance other phenomena such as nonlinear wave mixing and harmonic generation. Our results show how nanostructured media could be at the basis of the development of novel lasing sources with exceptional tunability, directionality, and efficiency while being compatible with plastic photonics CMOS and candidates for in-board interconnections for future computers.

\section{ACKNOWLEDGMENTS}

We thank M. Ibisate, C. Soukoulis, K. Sakoda, and J. J. Saenz for fruitful discussions. C.C. acknowledges the ERC (FP7/2007-2013) Grant No. 201766. R.S. acknowledges support by RyC, L.S.F.P. acknowledges support by JdC, and J.F.G.L. acknowledges support by JAE. The work was supported by EU FP7 NoE Nanophotonics4Enery Grant No. 248855; the Spanish MICINN CSD2007-0046 (Nanolight.es), MAT2009-07841 (GLUSFA) and Comunidad de Madrid S2009/MAT-1756 (PHAMA). 
[1] S. Gottardo, R. Sapienza, P. D. García, A. Blanco, D. S. Wiersma, and C. López, Nat. Phot. 2, 429 (2008); D. S. Wiersma, Nat. Phys. 4, 359 (2008).

[2] R. K. Chang and A. J. Campillo, Optical Processes in Microcavities (World Scientific, Singapore, 1996); K. L. van der Molen, P. Zijlstra, A. Lagendijk, and A. P. Mosk, Opt. Lett. 31, 1432 (2006).

[3] C. Conti and A. Fratalocchi, Nat. Phys. 4, 794 (2008).

[4] A. D. Stone, Phys. Scr. T 90, 248 (2001).

[5] C. López, Adv. Mater. 15, 1679 (2003).

[6] O. Painter et al., Science 284, 1819 (1999); H.-Y. Ryu, M. Notomi, E. Kuramoti, and T. Segawa, Appl. Phys. Lett. 84, 1067 (2004); H.-G. Park et al., Science 305, 1444 (2004).

[7] K. Yoshino, S. Tatsuhara, Y. Kawagishi, M. Ozaki, A. A. Zakhidov, and Z. V. Vardeny, Appl. Phys. Lett. 74, 2590 (1999); M. N. Shkunov, Z. V. Vardeny, M. C. DeLong, R. C. Polson, A. A. Zakhidov, and R. H. Baughman, Adv. Funct. Mater. 12, 21 (2002); Y. Nishijima, K. Ueno, S. Joudkazis, V. Mizeikis, H. Misawa, M. Maeda, and M. Minaki, Opt. Express 16, 13676 (2007); W. C. Stumpf, T. Asano, T. Kojima, M. Fujita, Y. Tanaka, and S. Noda, Phys. Rev. B 82, 075119 (2010).

[8] M. Scharrer, A. Yamilov, X. Wu, H. Cao, and R. P. H. Chang, Appl. Phys. Lett. 88, 201103 (2006).

[9] K. Sakoda, K. Ohtaka, and T. Ueta, Opt. Express 4, 481 (1999); J. P. Dowling, M. Scalora, M. J. Bloemer, and C. M. Bowden, J. Appl. Phys. 75, 1896 (1994).
[10] Y. Vlasov, K. Luterova, I. Pelant, B. Honerlage, and V. N. Astratov, Appl. Phys. Lett. 71, 1616 (1997).

[11] G. R. Maskali, M. A. Petruska, J. Nanda, I. V. Bezel, R. D. Schaller, H. Htoon, J. M. Pietryga, and V. I. Klimov, Adv. Mater. 18, 343 (2006).

[12] P. Jiang, J. F. Bertone, K. S. Hwang, and V. L. Colvin, Chem. Mater. 11, 2131 (1999).

[13] K. L. Shaklee and R. F. Leheny, Appl. Phys. Lett. 18, 475 (1971); L. Dal Negro, P. Bettotti, M. Cazzanelli, D. Pacifici, and L. Pavesi, Opt. Comm. 229, 337 (2004); L. Pavesi, L. Dal Negro, C. Mazzoleni, and G. F. F. Priolo, Nature (London) 408, 440 (2000); C. Lange, M. Schwalm, S. Chatterjee, and W. W. Rühle, Appl. Phys. Lett. 91, 1911071 (2007).

[14] M. Leonetti, R. Sapienza, M. Ibisate, C. Conti, and C. López, Opt. Lett. 34, 3764 (2009).

[15] S. G. Johnson and J. D. Joannopoulos, Opt. Express 8 (3), 173 (2001).

[16] K. Sakoda, Opt. Express 4 (5), 167 (1999); Optical Properties of Photonic Crystals, 2nd ed., Springer Series in Optical Sciences Vol. 80 (Springer, New York, 2001).

[17] R. Sapienza, P. D. García, J. Bertolotti, M. D. Martín, A. Blanco, L. Viña, C. López, and D. S. Wiersma, Phys. Rev. Lett. 99, 233902 (2007).

[18] P. D. García, R. Sapienza, L. S. Froufe-Pérez, and C. López, Phys. Rev. B 79, 241109 (2009). 